\title{
SOME COVERING AND EMBEDDING THEOREMS FOR INVERSE SEMIGROUPS
}

\author{
D. B. MCALLISTER
}

(Received 29 January 1975)

\begin{abstract}
An inverse semigroup $S$ is called $E$-unitary if the equations $e a=e=e^{2}$ together imply $a^{2}=a$. In a previous paper the author showed that every inverse semigroup is an idempotent separating homomorphic image of an $E$-unitary inverse semigroup. The main question considered in this paper is the following. Given an inverse semigroup $S$ give a method for constructing $E$-unitary inverse semigroups $P$ and idempotent separating homomorphisms $\phi: P \rightarrow S$ in such a way that the structure of $P$ as a $P$-semigroup is evident.
\end{abstract}

\section{Introduction}

We shall assume familiarity with the results and terminology of McAlister $(1974,1974 a)$. In these papers, $E$-unitary inverse semigroups were called proper inverse semigroups; the present terminology was suggested by $\mathrm{A}$. H. Clifford.

A one-to-one partial right translation of a semigroup $S$ is a one-to-one partial translation $\rho$ of $S$ such that $\Delta \rho$ is a left ideal of $S$ and $(x a) \rho=x(a \rho)$ for all $x \in S^{1}, a \in \Delta \rho$. The set $\hat{S}$ of all one-to-one partial right translations of $S$ is an inverse semigroup and it was shown by McAlister (1975) that, for inverse semigroups, the association $S \mapsto \hat{S}$ gives rise to a functor from the category of inverse semigroups to itself. The properties of this functor are discussed in Section 2. The semigroup $\hat{S}$ is calculated, in Section 3, for $S$ an $E$-unitary inverse semigroup in the form $P(G, \mathscr{X}, \mathscr{Y})$. This calculation is used to obtain an embedding of $S$ into the semidirect product of a semilattice by a group and to characterise the translational hull $\Omega(S)$ of $S$.

The main theorem of Section 4 shows that the forgetful functor, from the category of semidirect products of semilattices by groups and full homomorphisms, to the category of inverse semigroups and full homomorphisms, has an adjoint $\Gamma$. The structure of $\Gamma(S)$, for an inverse semigroup $S$, is described

This was partly supported by a National Science Foundation Grant. Portions of it were carried out while the author was visiting Monash University, Australia, May-July, 1974. 
explicitly in terms of $S$; likewise so is the projection homomorphism $\gamma_{s}: \Gamma(S) \rightarrow S$. This description can be used to give necessary and sufficient conditions for $S$ to be a homomorphic image of a semidirect product of a semilattice by a group and to characterise algebraically the semidirect product of a semilattice by a group.

An inverse semigroup $T$ is called a covering semigroup if $\gamma_{T}: \Gamma(T) \rightarrow T$ is onto. The results of Section 5 show that the problem of obtaining $E$-unitary covers $\phi: P \rightarrow S$ of an inverse semigroup $S$ by $E$-unitary inverse semigroups $P$ can be replaced by the problem of embedding $S$ in a covering semigroup $T$. Given such an embedding, the functors of Section 2 and 5 are used to obtain a covering $\gamma(T ; S): \Gamma(T ; S) \rightarrow S$ of $S$ by an $E$-unitary inverse semigroup $\Gamma(T, S)$. Further, it is shown that the $\Gamma(T ; S)$ form a cofinal subcategory of $E$-unitary coverings of $S$; in general, this category does not have a final object.

The last section of the paper consists of examples. The first of these describes all $E$-unitary covers of a Brandt semigroup $S$ and shows that, for such semigroups, the category of all $E$-unitary covers does not have a final object.

\section{One-to-one partial right translations of an inverse semigroup}

Definition 2.1. Let $S$ be a semigroup. Then a one-to-one partial right translation $\rho$ of $S$ is a one-to-one partial transformation of $S$ such that

(i) the domain $\Delta \rho$ of $\rho$ is a left ideal of $S$;

(ii) for each $a \in \Delta \rho, x \in S$, $(x a) \rho=x(a \rho)$.

The set $\hat{S}$ of all one-to-one partial right translations of a semigroup $S$ is an inverse semigroup under composition. It was studied, in detail, by McAlister (1975).

Definition 2.2. (Schein, 1973) $A$ pair of elements $a, b$ in an inverse semigroup $S$ is called compatible if $a b^{-1}$ and $a^{-1} b$ are both idempotents. $A$ set $A$ of elements of $S$ is compatible if each pair of elements of $A$ is compatible.

The importance of compatible elements stems from the fact that if elements $a, b$ in an inverse semigroup $S$ have an upper bound, under the usual partial ordering, in some inverse semigroup $T$, which contains $S$ as a subsemigroup, then $a$ and $b$ are compatible.

Definition 2.3. (Schein, 1973) An inverse semigroup $T$ is complete if each compatible subset $A$ of $T$ has a least upper bound VA in $T$.

$A$ homomorphism $\theta$ of an inverse semigroup $S$ into an inverse semigroup $T$ is complete if, for any subset $A$ of $S$,

$$
(V A) \theta=V(A \theta)
$$

whenever VA exists in $S$. 
$\hat{S}$ is a complete inverse semigroup for each semigroup $S$; if $S$ is an inverse semigroup then the Preston-Vagner theorem, Clifford and Preston, (1961), page 30 provides an embedding $\eta_{s}$ of $S$ into $\hat{S}$ : for each $a \in S, a \eta_{s}=\rho_{a}$ where

$$
\Delta \rho_{a}=S a a^{-1} \text { and } x \rho_{a}=x a \text { for each } x \in S a a^{-1} .
$$

THEOREM 2.4. Let $\theta$ be a homomorphism of an inverse semigroup $S$ into an inverse semigroup $T$ and for each $\rho \in \hat{S}$ define

$$
\rho \hat{\theta}=\left\{(t(a \theta), t(b) \theta) \in T \times T: t \in T^{1},(a, b) \in \rho\right\} .
$$

Then $\hat{\theta}: \rho \mapsto \rho \hat{\theta}$ is the unique complete homomorphism of $\hat{S}$ into $\hat{T}$ such that the diagram

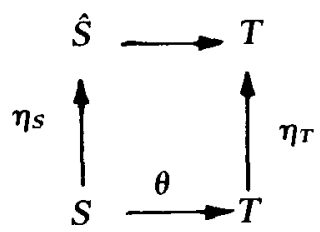

commutes.

Proof. It was shown in McAlister (1975) that $\hat{\theta}$ is a complete homomorphism of $\hat{S}$ into $\hat{T}$. The fact that the diagram commutes is a consequence of straightforward computation. While the uniqueness of $\hat{\theta}$ follows because any $\rho \in \hat{S}$ can be expressed as $V\left\{\rho_{e \rho}: e^{2}=e \in \Delta \rho\right\}$ where, as above, $\Delta \rho_{a}=S a a^{-1}$ and $x \rho_{a}=x a$ for each $x \in S a a^{-1}$.

If we consider $\rho$ as an operator on $S$ rather than as a relation on $S$ then the definition of $\rho \hat{\theta}$ can be rephrased as follows: $\rho \hat{\theta}=\rho^{*}$ where

$$
\Delta \rho^{*}=\left\{t \in T: t^{-1} t \leqq e \theta \text { for some } e^{2}=e \in \Delta \rho\right\}
$$

and

$$
t \rho^{*}=t(e \rho) \theta \text { if } t^{-1} t \leqq e \theta \text { where } e \in \Delta \rho .
$$

Because of this it is easy to see that the following corollaries hold.

COROLLARY 2.5. Let $S$ be a homomorphism of an inverse semigroup $S$ into an inverse semigroup $T$. If $\theta$ is idempotent separating so is $\hat{\theta}: \hat{S} \rightarrow \hat{T}$; if $\theta$ is a full homomorphism (i.e. So contains all the idempotents of $T$ ) then so is $\hat{\theta}$.

COROLlaRY 2.6. Let $S$ be an inverse semigroup with semilattice of idempotents $E$. Then the semilattice of idempotents of $\hat{S}$ is isomorphic to $\hat{E}$.

COROLlaRY 2.7. If $\theta$ is a full homomorphism of an inverse semigroup $S$ into an inverse semigroup $T$ then $\theta$ maps the group of units $\Sigma(S)$ of $\hat{S}$ into the group of units $\Sigma(T)$ of $T$. 
In a recent paper Schein, (1973), has shown that the translational hull $\Omega(S)$ of an inverse semigroup $S$ can be described as a semigroup of subsets of $S$. The semigroup $S$ can be described in a similar way.

Definition 2.8. (Schein, 1973). Let $S$ be an inverse semigroup. Then a subset $H$ of $S$ is called permissible if

(i) $a \in H, b \leqq a$ implies $b \in H$;

(ii) $a, b \in H$ implies $a b^{-1}, a^{-1} b$ idempotent.

THEOREM 2.9. Let $S$ be an inverse semigroup. Then $\hat{S}$ is isomorphic to the set of all permissible subsets of $S$ under subset multiplication.

Proof. For each $\rho \in \hat{S}$, let $H_{\rho}=\left\{e p: e^{2}=e \in \Delta \rho\right\}$ and let $\phi$ be the mapping of $\hat{S}$ into $2^{S}$ defined by $\rho \phi=H_{\rho}$ for each $\rho \in \hat{S}$. Then $\phi$ can be shown to be an isomorphism of $\hat{S}$ onto $\{H \subseteq S: H$ is permissible $\}$.

Schein (1973) has proved that the translational hull $\Omega(S)$ of an inverse semigroup $S$ can be identified with the idealiser in $\{H \subseteq S: H$ is permissible $\}$ of $\left\{H_{a}: a \in S\right\}$, where $H_{a}=\{x \in S: x \leqq a\}$. Under the isomorphism $\phi$ defined in Theorem 2.9, $H_{a}$ corresponds to the inner right translation $\rho_{a}=a \eta_{s}$ of $S$ with domain $S a a^{-1}$ and $x \rho_{a}=x a$ for each $x \in S a a^{-1}$. Hence we have,

Corollary 2.10. Let $S$ be an inverse semigroup. Then there is a unique isomorphism $\psi_{s}$ such that the following diagram commutes where $\pi_{s}$ is the one-to-one homomorphism of $S$ into $\Omega(S)$ defined by $a \pi_{S}=(\lambda, \rho)$ where $\lambda x=a x$, $x \rho=x a$ for each $x \in S$ and $I\left(S \eta_{s}\right)$ is the idealiser of $S \eta_{s}$ in $\hat{S}$.

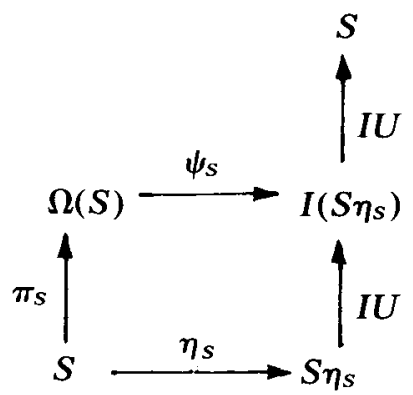

Lemma 2.11. Let $A, B, C, D$ be inverse semigroups with $C \subseteq A, D \subseteq B$ and let $\theta$ be a homomorphism of $A$ into $B$ such that $C \theta \subseteq D$. If $C \theta$ is a full inverse subsemigroup of $D$, then $I(C) \theta \subseteq I(D)$, where, for example, $I(D)$ denotes the idealiser of $D$ in $B$. If $\theta$ is also a full idempotent separating homomorphism of $A$ into $B$, then $I(C) \theta$ is a full inverse subsemigroup of $I(D)$.

PROOF. This is straightforward. 
If Lemma 2.11, Corollary 2.10 and Corollary 2.5 are combined, we obtain the following result which is essentially due to N. R. Reilly (1974).

Proposition 2.12. Let $\theta$ be a homomorphism of an inverse semigroup $S$ onto a full inverse subsemigroup of an inverse semigroup $T$. Then there is a unique complete homomorphism $\Omega(\theta)$ of $\Omega(T)$ such that $\theta \pi_{T}=\pi_{s} \Omega(\theta)$.

If $\theta$ is idempotent separating, then $\Omega(\theta)$ is idempotent separating and full.

COROLlary 2.13 (Ault, 1972). Let $S$ be an inverse semigroup with semilattice of idempotents $E$. Then the semilattice of idempotents of $\Omega(S)$ is isomorphic to $\Omega(E)$.

The intersection of a finite number of non-empty left ideals, of an inverse semigroup $S$, is non-empty. Because of this, the set $S^{*}$ of non-zero elements of $\hat{S}$ is an inverse subsemigroup. Further, if $\theta: S \rightarrow T$ is a homomorphism of $S$ into an inverse semigroup $T, \hat{\theta}$ maps $S^{*}$ into $T^{*}$. It follows that the analogs of Theorem 1.4-Corollary 1.7 hold with $\hat{S}$ replaced by $S^{*}$. We shall use these where necessary in the sequel.

Finally, the following lemma from McAlister (1975) is needed later in the paper.

Lemma 2.14. Let $S$ be an inverse semigroup and let $\alpha \in \hat{S}$. Then, for each $a \in \Delta \alpha, a \mathscr{R} a \alpha$.

\section{3. $E$-unitary inverse semigroups}

DEFINITION 3.1. An inverse semigroup $S$ is $E$-unitary if the equations ea $=$ $e=e^{2}$ together imply $a^{2}=a$ for all $a, e \in S$.

$E$-unitary inverse semigroups were introduced by Saito (1965), who called them proper inverse semigroups. The latter name was also used by McAlister (1974, 1974a). E-unitary inverse semigroups have also been called reduced inverse semigroups, O'Carroll (1974). The present terminology was suggested by A. H. Clifford.

Let $\mathscr{X}$ be a partially ordered set and let $G$ be a group which acts on $\mathscr{X}$, on the left, by order automorphisms. If $\mathscr{Y}$ is a subsemilattice of $\mathscr{X}$ then the set

$$
P(G, \mathscr{X}, \mathscr{Y})=\left\{(A, g) \in \mathscr{Y} \times G: A \wedge g B, g^{-1}(A \wedge B) \in \mathscr{Y} \text { for all } B \in \mathscr{Y}\right\}
$$

is an $E$-unitary inverse semigroup; McAlister, (1974), Lemma 1.1.

The following technical lemma shows that in some circumstances, the definition of $P(G, \mathscr{X}, \mathscr{Y})$ can be considerably simplified.

LemMA 3.2. Let $\mathscr{P}$ be a partially ordered set and let $G$ be a group which acts on $\mathscr{X}$ by order automorphisms; let $\mathscr{Y}$ be a subsemilattice of $\mathscr{X}$.

(A) Suppose that the following condition holds: 
if $A, B, g A \in \mathscr{Y}$, where $g \in G$ and $B \leqq A$, then $g B \in \mathscr{Y}$.

Then

$$
P(G, \mathscr{X}, \mathscr{Y})=\left\{(A, g) \in \mathscr{Y} \times G: g^{-1} A \in \mathscr{Y}\right\}
$$

(B) Suppose that the following condition holds:

Then

$$
\text { if } A, B \in \mathscr{Y} \text { and } g A \leqq B \text {, for some } g \in G \text {, then } g A \in \mathscr{Y} \text {. }
$$

$\mathscr{Y}$ is an ideal of $\mathscr{Z}=G \mathscr{Y}$ and the following are equivalent

(i) for each $g \in G, g \mathscr{Y} \cap \mathscr{Y} \neq \square$

and

(ii) for each $A \in \mathscr{Z}$ there exists $B \in \mathscr{Y}$ such that $A \geqq B$.

Further

$$
P(G, \mathscr{X}, \mathscr{Y})=P(G, \mathscr{X}, \mathscr{Y})=\left\{(A, g) \in \mathscr{Y} \times G: g^{-1} A \in \mathscr{Y}\right\} .
$$

Proof. (A) If $(A, g) \in P(G, \mathscr{X}, \mathscr{Y})$ then $g^{-1} A=g^{-1}(A \wedge A) \in \mathscr{Y}$ by definition. On the other hand, suppose that $A, g^{-1} A \in \mathscr{Y}$ and let $B \in \mathscr{Y}$. Then $A \wedge B \in \mathscr{Y}$ and $A \wedge B \leqq A$ so that, since $g^{-1} A \in \mathscr{Y}, g^{-1}(A \wedge B) \in \mathscr{Y}$. Further $g^{-1} A \wedge B \in \mathscr{Y}$ and $g^{-1} A \wedge B \leqq g^{-1} A \in \mathscr{Y}$ so that, since $A=g\left(g^{-1} A\right) \in \mathscr{Y}$, $A \wedge g B=g\left(g^{-1} A \wedge B\right) \in \mathscr{Y}$. Hence $(A, g) \in P(G, \mathscr{X}, \mathscr{Y})$.

(B) First, let $C \in \mathscr{L}$ and suppose $C \leqq A$ where $A \in \mathscr{Y}$. Then $C=g B$ for iome $g \in G, B \in \mathscr{Y}$. Hence, by the hypothesis in (B), $C \in \mathscr{Y}$. That is, $\mathscr{Y}$ is an deal of $\mathscr{X}$.

Now suppose (i) holds and let $A \in \mathscr{Z}$; then $A=g C$ for some $C \in \mathscr{Y}$. Let $D \in \mathscr{Y}$ be such that $g D \in \mathscr{Y}$, by (i), such a $D$ exists, and set $B=g(C \wedge D)$. Then $B=g(C \wedge D) \leqq g D \in \mathscr{Y}$ and $C \wedge D \in \mathscr{Y}$. Hence $B \in \mathscr{Y}$ and $B \leqq g C=A$.

Suppose (ii) holds and let $A \in \mathscr{Y}, g \in G$. Then $g^{-1} A \geqq B$ for some $B \in \mathscr{Y}$. This gives $g B \leqq A$ where $A, B \in \mathscr{Y}$. Hence, by the hypothesis in (B), $g B \in \mathscr{Y}$ so hat (i) holds.

Finally, it is clear that the hypothesis in (B) implies the hypothesis in (A) so hat $P(G, \mathscr{X}, \mathscr{Y})=\left\{(A, g) \in \mathscr{Y} \times G: g^{-1} A \in \mathscr{Y}\right\}=P(G, \mathscr{Z}, \mathscr{Y})$.

COROLlary 3.3 If $\mathscr{Y}$ is an ideal of $\mathscr{X}$, as well as a subsemilattice, then ${ }^{\mathrm{D}}(G, \mathscr{X}, \mathscr{Y})=\left\{(A, g) \in \mathscr{Y} \times G: g^{-1} A \in \mathscr{Y}\right\}$.

DefinItion 3.4. A partially ordered set $\mathscr{X}$ is a near semilattice if, for each $\mathcal{A} \in \mathscr{X}$, the set $\{X \in \mathscr{X}: X \leqq A\}$ is a subsemilattice of $\mathscr{X}$.

DEFINITION 3.5. An ideal $\mathscr{Y}$ of a partially ordered set $\mathscr{R}$ is essential if, for each $\mathcal{A} \in \mathscr{X}$, there exists $B \in \mathscr{Y}$ such that $B \leqq A$.

The main theorem of McAlister (1974a), (Theorem 2.6), can be stated as ollows. 
THEOREM 3.6. Let $\mathscr{X}$ be a near semilattice with 9 an essential ideal and subsemilattice of $\mathscr{X}$. Let $G$ be a group which acts on $\mathscr{X}$ by order automorphisms in such a way that $\mathscr{X}=G . \mathscr{Y}$. Then $P(G, \mathscr{X}, \mathscr{Y})$ is an $E$-unitary inverse semigroup.

Conversely, if $S$ is an $E$-unitary inverse semigroup, then $S \approx P(G, \mathscr{X}, \mathscr{Y})$ for some $G, \mathscr{X}, \mathscr{Y}$ as above, which are unique up to the equivalence of group actions.

In this section, we shall calculate the semigroup $\hat{S}$ for $S=P(G, \mathscr{X}, \mathscr{Y})$. Using this, we can obtain an explicit construction for $\Omega(S)$.

If $\mathscr{X}$ is a partially ordered set we shall denote by $\mathscr{X}^{*}$ the set of all non-empty order ideals of $\mathscr{X}$. If $\mathscr{Y}$ is an essential ideal and subsemilattice of $\mathscr{X}$ then, clearly, $\mathscr{Y}^{*}$ is an essential ideal and subsemilattice of $\mathscr{X}^{*}$. If a group $G$ acts on $\mathscr{X}$ by order automorphisms then $G$ acts on $\mathscr{X}^{*}$ by order automorphisms as follows

$$
g \cdot A=\{g \cdot a: a \in A\} \quad \text { for each } A \in \mathscr{X}^{*} .
$$

Proposition 3.7. Let $\mathscr{X}$ be a near semilattice with $y$ an essential ideal and subsemilattice of $\mathscr{X}$ and let $G$ be a group which acts on $\mathscr{X}$ by order automorphisms in such a way that $\mathscr{X}=G \cdot \mathscr{Y}$. Then, if $P=P(G, \mathscr{X}, \mathscr{Y})$, the nonzero elements of $\dot{F}$ form a semigroup $P^{*}$ isomorphic to $P\left(G, \mathscr{X}^{*}, \mathscr{Y}^{*}\right)$.

Proof. Let $\rho \in P^{*}$ and let $(A, 1) \in \Delta \rho$. Then $(\mathrm{A}, 1) \rho=\left(\mathrm{A}, \mathrm{g}_{\mathrm{A}}\right)$ for some $g_{A} \in G$ by Lemma 2.14. For any $B \in \mathscr{Y}$,

$$
\left(B \wedge A, g_{B \wedge A}\right)=(B \wedge A, 1) \rho=(B, 1)[(A, 1) \rho]=\left(B \wedge A, g_{A}\right)
$$

so that $g_{B \wedge A}=g_{A}$ for all $B \in \mathcal{Y}$. In particular, $g_{A}=g_{B}$ for all $B \in \mathscr{Y}$ such tha $(B, 1) \in \Delta \rho$. Hence there exists $g_{\rho} \in G$ such that, for each $(B, 1) \in \Delta \rho$,

$$
(B, 1) \rho=\left(B, g_{\rho}\right)
$$

Let $I_{\rho}=\{B \in \mathscr{Y}:(B, 1) \in \Delta \rho\}$. Then $I_{\rho}$ is an order ideal of $y$ and, sinc $\left(B, g_{\rho}\right) \in P$ for each $B \in I_{\rho}, g_{\rho}^{-1} I_{\rho} \subseteq \mathscr{Y}$. Hence the map $\psi$ defined by

$$
\rho \psi=\left(I_{\rho}, g_{\rho}\right)
$$

is a mapping of $P^{*}$ into $P\left(G, \mathscr{Z}^{*}, \mathscr{Y}^{*}\right)$. Since

$$
(B, h) \rho=(B, h)\left[\left(h^{-1} B, 1\right)\right] \rho=\left(B, h g_{\rho}\right)
$$

for each $(B, h) \in \Delta \rho, \psi$ is clearly one-to-one.

Suppose $\rho, \sigma \in P^{*}$. Then $(A, 1) \in \Delta \rho \sigma$ if and only if $A \in I_{\rho}$ and $\left(A, g_{\rho}\right)$, $\Delta \sigma$. Now $\left(A, g_{\rho}\right) \in \Delta \sigma$ if and only if $\left(g_{\rho}^{-1} A, 1\right) \in \Delta \sigma$ which occurs if and only $g_{\rho}^{-1} A \in I_{\sigma}$. Hence $I_{\rho \sigma}=I_{\rho} \cap g_{\rho} I_{\sigma}$. Further, for $(A, 1) \in \Delta \rho \sigma$,

$$
(A, 1) \rho \sigma=\left(A, g_{\rho}\right) \sigma=\left(A, g_{\rho}\right)\left[\left(g_{\rho}^{-1} A, 1\right) \sigma\right]=\left(A, g_{\rho} g_{\sigma}\right) .
$$

Thus $g_{\rho \sigma}=g_{\rho} g_{\sigma}$ and $\psi$ is a homomorphism of $P^{*}$ into $P\left(G, \mathscr{X}^{*}, \mathscr{Y}^{*}\right)$.

Suppose now that $(I, g) \in P\left(G, \mathscr{X}^{*}, \mathscr{Y}^{*}\right)$ and define $\rho$ by 


$$
\Delta \rho=\left\{(A, h) \in P: h^{-1} A \in I\right\} \text { with }(A, h) \rho=(A, h g) \text { for }(A, h) \in \Delta \rho .
$$

Then it is a straightforward matter to show that $\rho \in P^{*}$ and $\rho \psi=(I, g)$. This shows that $\psi$ is a one-to-one homomorphism of $P^{*}$ onto $P\left(G, \mathscr{X}^{*}, \mathscr{Y}^{*}\right)$. That is, $P^{*} \approx P\left(G, \mathscr{X}^{*}, \mathscr{Y}^{*}\right)$.

DEFINITION 3.8 (Reilly, 1974). Let $\mathscr{X}$ be a partially ordered set and let $I$ be an ideal of $\mathscr{X}$. Then $I$ is called a p-ideal of $\mathscr{X}$ if, for each $A \in \mathscr{X}$, the set $\{X \in I: X \leqq A\}$ is a principal ideal of $\mathscr{X}$; that is, if $\{X \in I: X \leqq A\}$ has a greatest element. We denote by $\overline{\mathscr{X}}$ the set of non-empty $p$-ideals of $\mathscr{X}$; if $\mathscr{X}$ is a semilattice, so is $\overline{\mathscr{X}}$.

TheOrem 3.9. Let $P=P(G, \mathscr{X}, \mathscr{Y})$ be an $E$-unitary inverse semigroup. Then

$$
\Omega(P) \approx P\left(G, \mathscr{X}^{*}, \overline{\mathscr{Y}}\right) .
$$

Proof. By Corollary 2.10, we need only show that the idealiser of $T=P \eta_{P} \psi$ in $P\left(G, \mathscr{X}^{*}, \mathscr{Y}^{*}\right)$ is $P\left(G, \mathscr{X}^{*}, \overline{\mathscr{Y}}\right)$.

For each $(A, g) \in P,(A, g) \eta_{P}$ has domain $\left\{(B, h): h^{-1} B \leqq A\right\}$ and for each $(B, 1) \in \Delta(A, g) \eta_{p}, \quad(B, 1)\left[(A, g) \eta_{p}\right]=(B, g), \quad$ Hence $\quad(A, g) \eta_{p} \psi=((\leftarrow, A], g)$ where $(\leftarrow, A]=\{B \in \mathscr{Y}: B \leqq A\}$ and $g^{-1}(\leftarrow, A]=\left(\leftarrow, g^{-1} A\right] \subseteq \mathscr{Y}$. Thus $T=$ $P\left(G, \mathscr{X}_{p}, \mathscr{Y}_{p}\right)$ where, for any partially ordered set $\mathscr{X}, \mathscr{X}_{p}$ denotes the set of principal ideals $(\leftarrow, A], A \in \mathscr{X}$, of $\mathscr{X}$.

Suppose $(\mathscr{B}, h) \in I(T)$. Then, since $I(T)$ is an inverse subsemigroup of $P\left(G, \mathscr{X}^{*}, \mathscr{Y}^{*}\right),(\mathscr{B}, 1) \in I(T)$ and $\left(h^{-1} \mathscr{B}, 1\right) \in I(T)$. Thus $(\mathscr{B}, 1)((\leftarrow, A], 1) \in T$ for each $A \in \mathscr{Y}$. That is, $\mathscr{B} \cap(\leftarrow, A]$ is a principal ideal of $\mathscr{Y}$ for each $A \in \mathscr{Y}$. Hence $\mathscr{B}$ is a $p$-ideal of $\mathscr{Y}$. Similarly, $h^{-1} \mathscr{B}$ is a $p$-ideal of $\mathscr{Y}$.

Conversely, let $(\mathscr{B}, h) \in P\left(G, \mathscr{X}^{*}, \mathscr{Y}^{*}\right)$ be such that $\mathscr{B}, h^{-1} \mathscr{B} \in \bar{y}$ and let $((\leftarrow, A], g) \in T$. Then

$$
\begin{aligned}
(\mathscr{B}, h)((\leftarrow, A], g) & =\left(h\left(h^{-1} \mathscr{B} \cap(\leftarrow, A]\right), h g\right) \\
& =(h(\leftarrow, B], h g)=((\leftarrow, h B], h g)
\end{aligned}
$$

for some $B \leqq A$, since $h^{-1} \mathscr{B}$ is a $p$-ideal of $\mathscr{Y}$. But $h B \in h\left(h^{-1} \mathscr{B}\right)=\mathscr{B} \subseteq \mathscr{Y}$ and $(h g)^{-1}(\leftarrow, h B]=g^{-1}(\leftarrow, B] \subseteq g^{-1}(\leftarrow, A] \subseteq \mathscr{Y}$ so that $((\leftarrow, h B], h g) \in T$. Similarly, $((\leftarrow, A], g)(\mathscr{B}, h) \in T$ because $\mathscr{B}$ is a $p$-ideal of $\mathscr{Y}$. Hence

$$
I(T)=\left\{(\mathscr{B}, h) \in \bar{y} \times G: h^{-1} \mathscr{B} \in \bar{y}\right\} .
$$

The $p$-ideals of $\mathscr{Y}$ satisfy the relations $\mathscr{B} \subseteq \mathscr{A}$, with $\mathscr{B}, \mathscr{A}, g \mathscr{A} \in \overline{\mathscr{Y}}$ imply $g \mathscr{B} \in \overline{\mathscr{Y}}$. Thus, by Lemma $3.2(\mathrm{~A}), I(T)=P\left(G, \mathscr{X}^{*}, \overline{\mathscr{Y}}\right)$.

COROllary 3.10. If $S$ is an E-unitary inverse semigroup, so are $S^{*}$ and $\Omega(S)$. 
Definition 3.11 (McFadden and O'Carroll, 1971). An inverse semigroup $S$ is called $F$-inverse if each class of the congruence $\sigma=\{(a, b) \in S \times S: e a=e b$ for some $e^{2}=e$ in $\left.S\right\}$ has a maximum member under the usual partial ordering on $S$.

It is shown in McAlister, (1974a), Theorem 2.8 that $F$-inverse semigroups are, up to isomorphism, the semigroups of the form $P(G, \mathscr{X}, \mathscr{Y})$ where $\mathscr{X}$ is a semilattice and $\mathscr{Y}$ is a principal ideal of $\mathscr{X}$.

Let $P=P(G, \mathscr{X}, \mathscr{Y})$ be an $E$-unitary inverse semigroup where $\mathscr{X}$ is a near semilattice, $\mathscr{Y}$ is an essential ideal and subsemilattice of $\mathscr{X}$. Then the intersection of any two non-empty order ideals of $\mathscr{X}$ is again non-empty. For, if $A, B \in \mathscr{X}^{*}$, there exist $a \in A \cap \mathscr{Y}, b \in B \cap \mathscr{Y}$ since $\mathscr{Y}$ is essential and then $a \wedge b \in A \cap B$. Hence $\mathscr{X}^{*}$ is a semilattice and we have

$$
P \subseteq P^{*}=P\left(G, \mathscr{X}^{*}, \mathscr{Y}^{*}\right) \subseteq P\left(G, \mathscr{X}^{*}, \mathscr{X}^{*}\right)
$$

where $P\left(G, \mathscr{X}^{*}, \mathscr{Y}^{*}\right)$ is $F$-inverse and $P\left(G, \mathscr{X}^{*}, \mathscr{X}^{*}\right)$ is a semidirect product of $\mathscr{X}^{*}$ by $G$.

Thus we have the following result which is due to O'Carroll (1975).

THEOREM 3.12. If $S$ is an E-unitary inverse semigroup then $S$ can be embedded in an F-inverse semigroup. Further $S$ can be embedded in the semidirect product of a semilattice by a group.

\section{Covering by semidirect products}

Let $S$ be an inverse semigroup. Then, by McAlister (1974a), Theorem 2.6 there is an $E$-unitary inverse semigroup $P$ and an idempotent separating homomorphism $\phi$ of $P$ onto $S$. It is a natural question to ask if there is a universal such $E$-unitary inverse semigroup $P$ and homomorphism $\phi$ of $P$ onto $S$. The answer, in general, is no; see Example 6.1 in Section 6. We shall show that, if the category of $E$-unitary inverse semigroups is replaced by the category of inverse semigroups which are semidirect products of semilattices by groups, and onto homomorphisms by full ones, the answer is yes. Further, the universal such object can be constructed in a natural manner from $S$ itself.

Lemma 4.1. Let $S$ be an inverse semigroup and let $\alpha \in \hat{S}$. Then, for each $e^{2}=e \in \nabla \alpha$,

$$
\alpha \rho_{\mathrm{e}} \alpha^{-1}=\rho_{\alpha \cdot \mathrm{e}}
$$

where

$$
\alpha \cdot e=\left(e \alpha^{-1}\right)^{-1}\left(e \alpha^{-1}\right) .
$$

Proof. Since $\alpha \rho_{e} \alpha^{-1}$ is an idempotent we need only calculate its domain, in order to evaluate it. Now, 
$x \in \Delta \alpha \rho_{e} \alpha^{-1} \Leftrightarrow x \in \Delta \alpha$ and $x \alpha \in S e$

$$
\begin{aligned}
& \Leftrightarrow x \in \Delta \alpha \text { and } x \in S e \alpha^{-1}=S\left(e \alpha^{-1}\right)^{-1}\left(e \alpha^{-1}\right) \text { since } e \in \nabla \alpha=\Delta \alpha^{-1} \\
& \Leftrightarrow x \in S(\alpha \cdot e) \text { since } \alpha \cdot e \in \Delta \alpha .
\end{aligned}
$$

Hence $\alpha \rho_{e} \alpha^{-1}=\rho_{\alpha \cdot e}$.

Corollary 4.2. Let $S$ be an inverse semigroup with semilatice of idempotents $E_{s}$. Then $\Sigma(S)$, the group of units of $\hat{S}$, acts on $E_{S}$ by order automorphisms as follows

$$
\alpha \cdot e=\left(e \alpha^{-1}\right)^{-1}\left(e \alpha^{-1}\right)
$$

Proof. By Lemma $4.1, \Sigma(S)$ acts as a group of automorphisms on the subsemigroup of idempotents $\left\{\rho_{e}: e^{2}=e \in E\right\}, E=E_{S}$, of $\hat{S}$. This action transfers directly to an action on $E$ as in the statement of the Corollary.

It follows from Corollary 4.2 that we can construct the semidirect product $\Gamma(S)=P\left(\Sigma(S), E_{S}, E_{S}\right)$ of $E_{S}$ by $\Sigma(S)$.

LEMMA 4.3. Let $S$ be an inverse semigroup with semilattice of idempotents $E$. Then the mapping $\gamma_{S}: \Gamma(S) \rightarrow S$ defined by

$$
(e, \alpha) \gamma_{s}=e \alpha
$$

is an idempotent separating homomorphism of $\Gamma(S)$ onto a full inverse subsemigroup of $S$.

Proof. We shall show that the mapping $\gamma^{*}: \Gamma(S) \rightarrow S \eta_{s}$ defined by

$$
(e, \alpha) \gamma^{*}=\rho_{e} \alpha=\rho_{e \alpha}
$$

is an idempotent separating homomorphism of $\Gamma(S)$ onto a full subsemigroup of $S \eta_{s}$. Then, since $\eta_{s}$ is an isomorphism, the result follows.

By definition,

$$
\begin{aligned}
(e, \alpha) \gamma_{.}^{*}(f, \beta) \gamma^{*} & =\rho_{e} \alpha \rho_{f} \beta=\rho_{e} \alpha \rho_{f} \alpha^{-1} \alpha \beta \\
& =\rho_{e} \rho_{\alpha \cdot f} \alpha \beta \quad \text { by Lemma 4.1 } \\
& =\rho_{(e \wedge \alpha \cdot f)} \alpha \beta=[(e, \alpha)(f, \beta)] \gamma^{*}
\end{aligned}
$$

Hence $\gamma^{*}$ is a homomorphism. Now $(e, 1) \gamma^{*}=\rho_{e}$ so that $\gamma^{*}$ is idempotent separating and full.

The results so far in this section give a semidirect product $\Gamma(S)$ for each inverse semigroup $S$. The next lemma shows that the correspondence is functorial. 
LEMMA 4.4. Let $S$ and $T$ be inverse semigroups and let $\theta$ be a homomorphism of $S$ onto a full inverse subsemigroup of $T$. Then there is a unique homomorphism $\Gamma(\theta)$ of $\Gamma(S)$ onto a full inverse subsemigroup of $\Gamma(T)$ such that the diagram

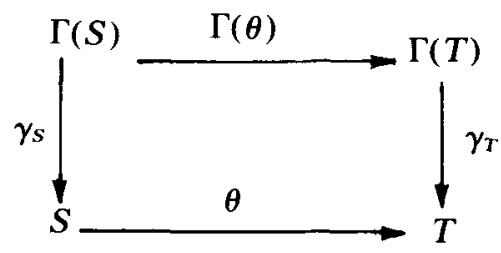

commutes.

Proof. Since $\theta$ maps $S$ onto a full inverse subsemigroup of $T$, it follows from Corollary 2.2 that $\hat{\theta}: \hat{S} \rightarrow \hat{T}$ is a full homomorphism. Hence $\hat{\theta}$ maps $\Sigma(S)$ into $\Sigma(T)$. Define $\Gamma(\theta)$ by

$$
(e, \alpha) \Gamma(\theta)=(e \theta, \alpha \hat{\theta}) .
$$

Then, by definition,

$$
(e, \alpha) \Gamma(\theta)(f, \beta) \Gamma(\theta)=(e \theta, \alpha \hat{\theta})(f \theta, \beta \hat{\theta})=(e \theta \wedge \alpha \hat{\theta} \cdot f \theta,(\alpha \beta) \hat{\theta}) .
$$

By the definition of the action of $\Sigma(T)$ on the idempotents of $T$,

$$
\begin{aligned}
\rho_{\alpha \hat{\theta} \cdot f \theta} & =\alpha \hat{\theta} \rho_{f \theta}(\alpha \hat{\theta})^{-1}=\alpha \hat{\theta} \rho_{f} \hat{\theta}(\alpha \hat{\theta})^{-1}=\left(\alpha \rho_{f} \alpha^{-1}\right) \hat{\theta} \\
& =\left(\rho_{\alpha \cdot f}\right) \hat{\theta}=\rho_{(\alpha \cdot f) \theta},
\end{aligned}
$$

so that $\alpha \hat{\theta} \cdot f \theta=(\alpha \cdot f) \theta$.

Hence

$$
\begin{aligned}
(e, \alpha) \Gamma(\theta)(f, \beta) \Gamma(\theta) & =(e \theta \wedge(\alpha \cdot f) \theta,(\alpha \beta) \hat{\theta})=(e \wedge \alpha \cdot f, \alpha \beta) \Gamma(\theta) \\
& =[(e, \alpha)(f, \beta)] \Gamma(\theta)
\end{aligned}
$$

so that $\Gamma(\theta)$ is a homomorphism of $\Gamma(S)$ into $\Gamma(T)$.

Further

$$
(e, \alpha) \Gamma(\theta) \gamma_{T}=(e \theta, \alpha \hat{\theta}) \gamma_{T}=(e \theta) \alpha \hat{\theta}=(e \alpha) \theta=(e, \alpha) \gamma_{D} \theta
$$

by the definition of $\alpha \hat{\theta}$, so that the diagram commutes. Finally, since $\gamma_{s} \theta$ is full and $\gamma_{T}$ is idempotent separating, $\Gamma(\theta)$ must be full.

To show that $\Gamma(\theta)$ is the unique homomorphism $\Gamma(S) \rightarrow \Gamma(T)$ which makes the diagram commute, suppose that $\psi: \Gamma(S) \rightarrow \Gamma(T)$ is such that $\psi \gamma_{T}=\gamma_{s} \theta$. Then, by McAlister (1975), Theorem 6.1, there is a homomorphism $\psi_{1}: \Sigma(S) \rightarrow \Sigma(T)$ and a homomorphism $\psi_{2}: E(S) \rightarrow E(T)$ such that 


$$
(e, \alpha) \psi=\left(e \psi_{2}, \alpha \psi_{1}\right) \quad \text { for all } \quad(e, \alpha) \in \Gamma(S) .
$$

Then $e \theta=(e, 1) \gamma_{S} \theta=(e, 1) \psi \gamma_{T}=\left(e \psi_{2}, 1\right) \gamma_{T}=e \psi_{2}$ for all $e \in E(S)$; thus $\psi_{2}=\theta$. Further

$$
(e \theta)\left(\alpha \psi_{1}\right)=\left(e \psi_{2}\right)\left(\alpha \psi_{1}\right)=(e, \alpha) \psi \gamma_{T}=(e, \alpha) \gamma_{s} \theta=(e \alpha) \theta=(e \theta)(\alpha \hat{\theta})
$$

for each $e \in E(S)$. Hence, since $\theta$ is full, $f\left(\alpha \psi_{1}\right)=f(\alpha \hat{\theta})$ for each idempotent $f \in T$. Let $t \in T$, then, since $\alpha \psi_{1}, \alpha \hat{\theta} \in \Sigma(T)$,

$$
t\left(\alpha \psi_{1}\right)=t\left[\left(t^{-1} t\right)\left(\alpha \psi_{t}\right)\right]=t\left[\left(t^{-1} t\right)(\alpha \hat{\theta})\right]=t(\alpha \hat{\theta}) .
$$

Hence $\alpha \psi_{1}=\alpha \hat{\theta}$ for all $\alpha \in \Sigma(S)$ and so $\psi=\Gamma(\theta)$.

Because of the uniqueness of $\Gamma(\theta), \Gamma$ preserves identities and composites. Thus we have the following theorem.

THEOREM 4.5. The mapping which associates with each inverse semigroup $S$ the semigroup $\Gamma(S)$ and, with each full homomorphism $\theta: S \rightarrow T$, the full homomorphism $\Gamma(\theta): \Gamma(S) \rightarrow \Gamma(T)$ is a functor from the category $\mathscr{I}$ of inverse semigroups and full homomorphisms to the category $\mathscr{P} \mathscr{D}$ of semidirect products of semilattices by groups, and full homomorphisms. The maps $\left\{\gamma_{S}: S \in \mathscr{I}\right\}$ form a natural transformation from $\Gamma$ to the identity functor on $\mathscr{I}$.

The functor $\Gamma$ is the adjoint to the forgetful functor $\mathscr{S} \mathscr{D} \rightarrow \mathscr{I}$. This follows from the next result which describes $\Gamma(S)$ for a proper inverse semigroup $S$.

LEMMA 4.6. Let $S=P(G, \mathscr{X}, \mathscr{Y})$ be an E-unitary inverse semigroup and let $H=\{g \in G: g \mathscr{Y}=\mathscr{Y}\}$. Then $\Gamma(S) \approx P(H, \mathscr{Y}, \mathscr{Y})$ where the action of $H$ on $\mathscr{Y}$ is inherited from that of $G$.

COROllary 4.7. If $S$ is a semidirect product of a semilattice by a group, then $\gamma_{S}: \Gamma(S) \approx S$.

THEOREM 4.8. Let $S$ be an inverse semigroup and let $T$ be a semidirect product of a semilattice by a group. If $\theta$ is a homomorphism of $T$ onto a full inverse subsemigroup of $S$, then there is a unique full homomorphism $\psi$ of $T$ into $\Gamma(S)$ such that the diagram

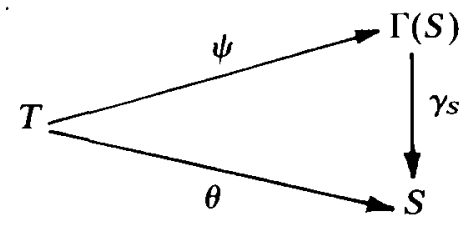

commutes.

Proof. By Lemma 4.4, $\Gamma(\theta)$ is the unique homomorphism $\Gamma(T) \rightarrow \Gamma(S)$ 
such that $\Gamma(\theta) \gamma_{S}=\gamma_{T} \theta$. Hence, since, by Corollary 4.7, $\gamma_{T}$ is an isomorphism, $\gamma_{T}^{-1} \Gamma(\theta)=\psi$ is the unique homomorphism such that $\theta=\psi \gamma_{s}$.

Corollary 4.9. Let $S$ be an inverse semigroup. Then the following statements are equivalent:

(1) S is a homomorphic image of the semidirect product of a semilattice by a group ;

(2) $\gamma_{s}$ is onto;

(3) each inner right translation of $S$ can be extended to a one-to-one right translation of $S$ ontoS;

(4) each element of $S$ belongs to some full permissible subset $H$ of $S$; $H$ is full if it meets each $\mathscr{L}$ and $\mathscr{R}$ class of $S$.

Proof $(1) \Leftrightarrow(2)$. Suppose there is a homomorphism $\theta$ of $T \in \mathscr{P D}$ onto $S$. Then by Theorem 4.8 there is a homomorphism $\psi: T \rightarrow \Gamma(S)$ such that $\theta=\psi \gamma_{s}$. Since $\theta$ is onto $\gamma_{s}$ must also be onto. The converse is immediate.

(2) $\Rightarrow$ (3). Let $a \in S$. Then $a=(e, \alpha) \gamma_{s}=e \alpha$ for some $e \in E(S), \alpha \in \Sigma(S)$. Since $(e, \alpha)(e, \alpha)^{-1}=(e, 1)$, it follows that $e=a a^{-1}$. Let $\rho=\rho_{e} \alpha$. Then $\rho$ has domain $S e=S a a^{-1}$ and $x \rho=x\left(a a^{-1}\right) \alpha=x a$ for each $x \in S a a^{-1}$. Hence $\rho_{a}=$ $\rho=\rho_{e} \alpha \leqq \alpha$.

(3) $\Leftrightarrow(4)$. This is immediate from Theorem 2.6 , since it is easy to see that full permissible subsets correspond to one-to-one right transformations of $S$ onto $S$.

(3) $\Rightarrow$ (2). Let $a \in S$ and let $\alpha \in \Sigma(S)$ extend $\rho_{a}$. Then

$$
\left(a a^{-1}, \alpha\right) \gamma_{s}=a a^{-1} \alpha=a a^{-1} \rho_{a}=a
$$

since $\alpha$ extends $\rho_{a}$. Hence $\gamma_{s}$ is onto.

Although Corollary 4.9 gives necessary and sufficient conditions on an inverse semigroup in order for it to be a homomorphic image of a semigroup in $\mathscr{P Q D}$, these are, in general, hard to verify. The result does nowever give some more amenable necessary conditions which, in certain situations, also turn out to be sufficient.

Proposition 4.10. Let $S$ be an inverse semigroup and suppose that $\gamma_{s}$ is onto. Then

(1) for each $a, e^{2}=e \in S$ there exist $b \in S$ such that $b b^{-1}=e$ and $e a=a a^{-1} b$ and (2) if e, f are $\mathscr{D}$-equivalent idempotents then $\{g \in E(S): g \geqq e\}$ is isomorphic to $\{g \in E(S): g \geqq f\}$.

Proof. (1) Let $\alpha \in \Sigma(S)$ extend $\rho_{a}$ and let $b=e \alpha$. Then, by Corollary 1.11 , $b b^{-1}=e \quad$ and $\quad\left(b b^{-1} a a^{-1}\right) \alpha=b b^{-1}\left(a a^{-1}\right) \rho_{a}=b b^{-1} a \quad$ while $\quad\left(a a^{-1} b b^{-1}\right) \alpha=$ $a a^{-1}\left(b b^{-1}\right) \alpha=a a^{-1} b$. Thus $a a^{-1} b=e a$. 
(2) Let $e \mathscr{D f}$. Then there exists $a \in S$ such that $a a^{-1}=e, a^{-1} a=f$. Suppose that $\alpha \in \Sigma(S)$ extends $\rho_{a}$. Then, by Corollary 4.2 the mapping $\phi: E_{S} \rightarrow E_{S}$ defined by $e \phi=\alpha^{-1} \cdot e$ is an order automorphism of $E(S)$, and so maps $\{g \in E(S): g \geqq e\}$ onto $\{g \in E(S): g \geqq e \phi\}$. But $e \phi=(e \alpha)^{-1}(e \alpha)=a^{-1} a=f$ since $\alpha$ extends $\rho_{a}$.

Proposition 4.11. Let $S=S^{1}$ be an inverse semigroup. Then $S$ is a homomorphic image of the semidirect product of a semilattice by a group if and only if for each $a \in S$ there exists $b \in S$ such that $b b^{-1}=1$ and $a=a a^{-1} b$.

Proof. By Proposition 4.10, the condition is necessary. Conversely, suppose that it is satisfied and let $b$ be a right unit of $S$. Then, by hypothesis, there is a right unit $c \in S$ such that $b^{-1}=b^{-1} b c$; that is $1=b c$. This implies $c=b^{-1}$ is a right unit so each right unit is invertible.

Now let $a \in S$ and let $b$ be a (right) unit such that $a=a a^{-1} b$. Then it is a straightforward matter to verify that $\rho_{b} \in \Sigma(S)$ and extends $\rho_{a}$. Thus, by Corollary 4.9 , the condition is sufficient.

The final application of Proposition 4.10 gives a characterisation of the semi-direct product of a semilattice by a group.

THEOREM 4.12. Let $S$ be an inverse semigroup then $S$ is isomorphic to the semidirect product of a semilattice by a group if and only if

(1) $S$ is E-unitary

and (2) for all $a \in S, e \in E(S)$ there exists $b \in S$ such that $b b^{-1}=e$, ea $=$ $a a^{-1} b$.

Proof. By Proposition 4.10, the conditions are clearly necessary. Conversely, suppose they are satisfied. Then, by McAlister (1974a), Theorem 2.6, we may suppose $S=P(G, \mathscr{X}, \mathscr{Y})$ for some $G, \mathscr{X}, \mathscr{Y}$ such that $\mathscr{Y} \cap g \mathscr{Y} \neq \square$ for each $g \in G$.

Let $A \in \mathscr{Y}, g \in G$. Then there exists $B \in \mathscr{Y}$ such that $g^{-1} B \in \mathscr{Y}$; that is $(B, g) \in S$. By (2), there exists $(C, h) \in S$ such that $(C, h)(C, h)^{-1}=(A, 1)$ and $(A, 1)(B, g)=(B, g)(B, g)^{-1}(C, h)$. These equations imply $C=A$ and $h=g$. Hence $(A, g) \in S$ and $S=P(G, \mathscr{Y}, \mathscr{Y})$. That is, $S$ is a semidirect product of $\mathscr{Y}$ by G.

Chen and Hsieh (1974), define an inverse semigroup $S$ to be factorizable if and only if there is a subgroup $G$ and a set $E$ of idempotent $E$ such that $S=G E$. They show that any factorizable inverse semigroup has an identity and it is easy to see that factorizable inverse semigroups are exactly these inverse monoids $S$ for which $\gamma_{s}: \Gamma(S) \rightarrow S$ is onto. 


\section{Covering and embedding theorems}

Let $S$ be an inverse semigroup and suppose that $S$ can be embedded in an inverse semigroup $T$ which is a homomorphic image of a semidirect product of a semilattice by a group. Then, by Corollary $4.7, \gamma_{T}$ is an idempotent separating homomorphism of $\Gamma(T)$ onto $T$. Thus $P=S \gamma_{T}^{-1}$ is an $E$-unitary inverse semigroup and the restriction of $\gamma_{T}$ to $P$ is an idempotent separating homomorphism of $P$ onto $S$. In general, however, $P$ does not appear directly in the form $P(G, \mathscr{X}, \mathscr{Y})$ with $\mathscr{Y})$ with $\mathscr{Y}$ an essential ideal and subsemilattice of $\mathscr{X}$. In this section we characterise those embeddings of $S$ which give rise in a natural manner to proper coverings $P(G, \mathscr{X}, \mathscr{Y})$ of $S$.

Definition 5.1. An inverse semigroup $S$ is a covering inverse semigroup if each inner right translation of $S$ can be extended to a member of $\Sigma(S)$.

The reason for this choice of name will become apparent later in the paper.

LEMMA 5.2. Let $S$ be an inverse semigroup and let $F$ be a subsemilattice of the semilattice $E$ of idempotents of $S$. Then

$$
\left\{a \in S:\left\{a a^{-1}, a^{-1} a\right\} \cup a F a^{-1} \cup a^{-1} F a \subseteq F\right\}
$$

is the largest inverse subsemigroup of $S$ with $F$ as its set of idempotents.

Proof. This is well known.

LEMMA 5.3. Let $S$ be an inverse semigroup and let $F$ be a subsemilattice of the semilattice $E$ of idempotents of $S$. Suppose that $F$ has the following property:

$$
\text { if } e \leqq f \text { and } e \mathscr{D} g \text { where } e \in E, f g \in F \text { then } e \in F \text {. }
$$

Then

$$
\left\{a \in S: a a^{-1}, a^{-1} a \in F\right\}
$$

is the largest inverse subsemigroup of $S$ with $F$ as its set of idempotents.

Proof. Set $S(F)=\left\{a \in S:\left\{a a^{-1}, a^{-1} a\right\} \cup a F a^{-1} \cup a^{-1} F a \subseteq F\right\}$. Then $a \in S(F)$ implies $a a^{-1}, a^{-1} a \in F$. On the other hand, suppose that $a a^{-1}, a^{-1} a \in$ $F$ and let $f \in F$. Then $a^{-1} f a \leqq a^{-1} a$ and $a^{-1} f a \mathscr{D} a\left(a^{-1} f a\right) a^{-1}=a a^{-1} f$. But $a a^{-1} f$, $a^{-1} a \in F$ since $F$ is a subsemilattice of $E$. Thus $a^{-1} f a \in F$. Similarly af $a^{-1} \in F$ so that $a \in S(F)$. Hence $S(F)=\left\{a \in S: a a^{-1}, a^{-1} a \in F\right\}$ so that, by Lemma 4.1, the result is proven.

COROLlary 5.4. Let $S$ be an inverse semigroup and let $F$ be an ideal of the semilattice of idempotents of $S$. Then $S(F)=\left\{a \in S: a a^{-1}, a^{-1} a \in F\right\}$.

DEFINITION 5.5 Let $S$ be an inverse subsemigroup of an inverse semigroup $T$. Then $S$ is a thick inverse subsemigroup of $T$ if 
(i) $e \leqq f$, e $\mathscr{D}_{\mathrm{r} g}$ with $f, g \in E_{S}, e \in E_{T}$ implies $e \in E_{s}$;

and (ii) $a a^{-1}, a^{-1} a \in E_{s}$ implies $a \in S$.

The inverse subsemigroup $S$ of $T$ is heavy if

(i)' $E_{S}$ is an ideal of $E_{T}$;

and (ii) $a a^{-1}, a^{-1} a \in E_{s}$ implies $a \in S$.

Clearly, every heavy inverse subsemigroup of $T$ is a thick subsemigroup of $T$.

The importance of Definition 5.5, for our purposes, is indicated by the following propositions.

Proposition 5.6. Let $\mathscr{X}$ be a semilattice and let $G$ be a group which acts on $\mathscr{X}$ by (order) automorphisms and let $T$ be the semidirect product of $\mathscr{Z}$ by $G$. Suppose that $S$ is an inverse subsemigroup of $T$ with semilattice of idempotents $\mathscr{Y} \times\{1\}$, where $\mathscr{Y}$ is a subsemilattice of $\mathscr{X}$.

If $S$ is a heavy subsemigroup of $T$, then $S=P(G, \mathscr{X}, \mathscr{Y})$.

If $S$ is a thick subsemigroup of $T$ then $\mathscr{Z}=G \cdot Y$ contains $\mathscr{Y}$ as an ideal and $S=P(G, \mathscr{Z}, \mathscr{Y})$.

Proof. The results follow in a straightforward manner using the definitions and Lemma 3.2.

Let $S$ be an inverse subsemigroup of a covering inverse semigroup $T$. Then we shall denote by $\Gamma(T ; S)$ the inverse subsemigroup $S \gamma_{T}^{-1}$ of $\Gamma(T)=P\left(\Sigma(T), E_{T}\right.$, $\left.E_{T}\right)$; we shall use $\gamma(T ; S)$ to denote the restriction of $\gamma_{T}$ to $\Gamma(T ; S)$.

Proposition 5.7. Let $S$ be an inverse subsemigroup of a covering inverse semigroup $T$. If $S$ is a thick (heavy) inverse subsemigroup of $T$ then $\Gamma(T ; S)$ is a thick (heavy) inverse subsemigroup of $\Gamma(T)$.

Proof. We prove the result for the case in which $S$ is a thick inverse subsemigroup of $T$. Because $\gamma_{T}$ is idempotent separating, the idempotents of $\Gamma(T ; S)$ are of the form $(f, 1)$ where $f \in E_{s}$. Suppose that $(e, 1) \leqq(f, 1)$ and $(e, 1) \mathscr{D}_{T}(g, 1)$ where $f, g \in E_{s}$. Then $e \leqq f$ and $e=\alpha^{-1} \cdot g$ for some $\alpha \in \Sigma(T)$. Now $\alpha^{-1} \cdot g=(g \alpha)^{-1} g \alpha$ and by Lemma $2.14, g=g \alpha(g \alpha)^{-1}$ so that, $g \mathscr{D}_{T} e$. Hence, since $S$ is a thick inverse subsemigroup of $T, e \in E_{S}$.

Let $(e, \alpha) \in \Gamma(T)$ and suppose that $(e, \alpha)(e, \alpha)^{-1}=(e, 1)$ and $(e, \alpha)^{-1}(e, \alpha)=$ $\left(\alpha^{-1} \cdot e, 1\right)$ are idempotents in $\Gamma(T ; S)$. Then, as above, $e=e \alpha(e \alpha)^{-1}, \alpha^{-1} \cdot e=$ $(e \alpha)^{-1}(e \alpha)$ so that, since $S$ is a thick inverse subsemigroup of $T$, ex $\in S$. This means that $(e, \alpha) \in \Gamma(T ; S)$.

The results of the first two paragraphs, combined, show that $\Gamma(T ; S)$ is a thick subsemigroup of $\Gamma(T)$.

Propositions 5.6 and 5.7 show that, if $S$ is an inverse semigroup, each embedding of $S$, as a thick (heavy) inverse semigroup of a covering inverse 
semigroup $T$, gives rise to an $E$-unitary inverse semigroup $\Gamma(T ; S)=P(G, \mathscr{X}, \mathscr{Y})$, with $\mathscr{Y}$ an ideal of $\mathscr{X}$ and $\mathscr{X}=G \cdot \mathscr{Y}$, and an idempotent separating homomorphism $\gamma(T ; S)$ of $\Gamma(T ; S)$ onto $S$. We shall show, conversely, that each covering of $S$ by an $E$-unitary inverse semigroup $P$ gives rise to an embedding of $S$ as a thick (heavy) inverse subsemigroup of a covering inverse semigroup $T$.

LeMMA 5.8. Let $\mathscr{Z}$ be a semilattice and let $\mathscr{Y}$ be a non-empty ideal of $\mathscr{X}$. Let $G$ be a group which acts on $\mathscr{Z}$ by order automorphisms in such a way that $\mathscr{Z}=G \mathscr{Y}$. Then each idempotent separating congruence on $P(G, \mathscr{X}, \mathscr{Y})$ can be uniquely extended to an idempotent separating congrence on $P(G, \mathscr{X}, \mathscr{X})$.

Proof. Let $\rho$ be an idempotent separating congruence on $P=P(G, \mathscr{X}, \mathscr{Y})$ and, for each $A \in \mathscr{Y}$, let $N_{A}=\{g \in G:(A, g) \rho(A, 1)\}$. Then $N_{A}$ is a subgroup of $G$ and

$$
(A, g) \rho(B, h) \text { if and only if } A=B \text { and } g h^{-1} \in N_{A} \text {. }
$$

Further, by McAlister (1974a), Theorem 3.3, the subgroups $N_{A}, A \in \mathscr{Y}$ obey the following conditions:

(i) $N_{A} \subseteq C_{A}=\{g \in G: g \cdot C$ for all $C \leqq A\}$

(ii) $A \geqq B$ implies $N_{A} \subseteq N_{B}$;

(iii) if $B=g \cdot A \in \mathscr{Y}$, where $A \in \mathscr{Y}$, then $N_{B}=g N_{A} g^{-1}$.

For each $D \in \mathscr{X}$, put $N_{D}=g N_{A} g^{-1}$ if $D=g \cdot A, A \in \mathscr{Y}$. Then $D=g \cdot A=h \cdot B$ implies $B=h^{-1} g \cdot A \in \mathscr{Y}$ which, by (iii), gives $N_{B}=h^{-1} g N_{A} g^{-1} h$; that is, $h N_{B} h^{-1}=g N_{A} g^{-1}$. Hence, since $\mathscr{Z}=G \cdot \mathscr{Y}$, the assignment $D \mapsto N_{D}$ is a well defined mapping of $\mathscr{X}$ into the lattice of subgroups of $G$.

It is a straightforward matter to show that the subgroups $\left\{N_{D}: D \in \mathscr{X}\right\}$ obey the analogs of (i), (ii), (iii) above. Hence, by McAlister (1974a), Theorem 3.3, the relation $\bar{\rho}$ on $P(G, \mathscr{X}, \mathscr{X})$ defined by

$$
(A, g) \bar{\rho}(B, h) \text { if and only if } A=B \text { and } g h^{-1} \in N_{A}
$$

is an idempotent separating congruence which, clearly, extends $\rho$.

Conversely, if $\rho^{\prime}$ is an idempotent separating congruence on $P(G, \mathscr{X}, \mathscr{X})$, which extends $\rho$, then it follows, from (iii) for $\rho^{\prime}$, that $\rho=\rho^{\prime}$.

Remark. Suppose that $\mathscr{X}, \mathscr{U}$ are semilattices with $\mathscr{y}, \mathscr{V}$ ideals of $\mathscr{X}, \mathscr{U}$ respectively, and let $G, H$ be groups acting on $\mathscr{X}, \mathscr{U}$ by order automorphisms in such a way that $\mathscr{X}=G \cdot \mathscr{Y}, \mathscr{U}=\boldsymbol{H} \cdot \mathscr{V}$. Then Lemma 4.8 shows that any idempotent separating homomorphism $\theta: P(G, \mathscr{X}, \mathscr{Y}) \rightarrow P(H, \mathscr{U}, \mathscr{V})$ can be extended to a homomorphism with domain $P(G, \mathscr{X}, \mathscr{X})$. It may not be possible to extend $\theta$ to a homomorphism $P(G, \mathscr{X}, \mathscr{X}) \rightarrow P(H, \mathscr{U}, \mathscr{U})$; see Example 6.2.

We can use Lemma 5.8 to prove a converse to Proposition 5.7 for $E$-unitary 
coverings of $S$ of the form $P(G, \mathscr{X}, \mathscr{Y})$ with $\mathscr{X}$ a semilattice and $\mathscr{Y}$ an ideal of $\mathscr{X}$. Note that if $S$ is a heavy subsemigroup $T$, then $\Gamma(T ; S)=P\left(\Sigma(T), E_{T}, E_{S}\right)$ is of the form above.

LEMMA 5.9. Let $\theta$ be an idempotent separating homomorphism of an inverse semigroup $T$ onto an inverse semigroup $\mathcal{U}$. If $S$ is a thick (heavy) inverse subsemigroup of $T$ then $S \theta$ is a thick (heavy) inverse subsemigroup of $\mathcal{U}$.

Proof. This is straightforward.

Proposition 5.10. Let $\mathscr{X}$ be a semilattice with $\mathscr{Y}$ an ideal of $\mathscr{X}$ and let $G$ be a group which acts on $\mathscr{X}$ by order automorphisms in such a way that $\mathscr{X}=G \cdot Y$. Suppose that $\phi$ is an idempotent separating homomorphism of $P(G, \mathscr{X}, \mathscr{Y})$ onto an inverse semigroup $S$. Then there is a covering semigroup $T$, containing $S$ as a heavy subsemigroup, and an idempotent separating homomorphism $\psi: P(G, \mathscr{X}, \mathscr{Y}) \rightarrow \Gamma(T ; S)$ such that the diagram

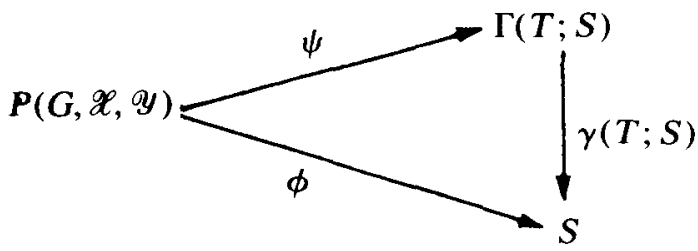

commutes.

Proof. Let $\rho=\phi \circ \phi^{-1}$; then, by Lemma 5.8, $\rho$ can be extended to an idempotent separating congruence $\bar{\rho}$ on $P(G, \mathscr{X}, \mathscr{X})$. Let $T=P(G ; \mathscr{X}, \mathscr{X}) / \bar{\rho}$ and denote by $\theta$ the natural homomorphism $P(G ; \mathscr{X}, \mathscr{X}) \rightarrow T$. By Lemma 5.9, $S=P(G, \mathscr{X}, \mathscr{Y}) \theta$ is a heavy inverse subsemigroup of $T$ because $P(G, \mathscr{X}, \mathscr{Y})$ is a heavy inverse subsemigroup of $P(G, \mathscr{X}, \mathscr{X})$.

By Theorem 4.8, there is a unique idempotent separating homomorphism $X: P(G, \mathscr{H}, \mathscr{X}) \rightarrow \Gamma(T)$ such that $\theta=X \gamma_{T}$. Since $P(G, \mathscr{X}, \mathscr{Y}) X \gamma_{T}=$ $P(G, \mathscr{X}, \mathscr{Y}) \theta=S$ we find that $P(G, \mathscr{X}, \mathscr{Y}) X \subseteq S \gamma_{r}^{-1}=\Gamma(T ; S)$ so that the restriction $\psi$ of $X$ to $P(G, \mathscr{X}, \mathscr{Y})$ is a homomorphism of $P(G, \mathscr{X}, \mathscr{Y})$ into $\Gamma(T ; S)$ such that $\psi \gamma(T ; S)=\phi$.

The general situation, involving arbitrary $E$-unitary inverse semigroups, is dealt with by combining Proposition 5.10 with the results of Sections 2 and 3 .

LEMMA 5.11. Let $\mathscr{X}$ be a partially ordered set with $\mathscr{Y}$ an essential ideal and subsemilattice of $\mathscr{X}$ and let $G$ be a group which acts on $\mathscr{X}$ by order automorphisms in such a way that $\mathscr{X}=G \cdot \mathscr{Y}$. Then $P(G, \mathscr{X}, \mathscr{Y})$ is a thick inverse subsemigroup of $P\left(G, \mathscr{X}^{* *}, \mathscr{X}^{*}\right)$ where $\mathscr{X}^{*}$ is the semilattice of non-empty order ideals of $\mathscr{X}$.

Proof. This is straightforward. 
Proposition 5.12. Let $P$ be an E-unitary inverse semigroup and let $\phi$ be an idempotent separating homomorphism of $P$ onto an inverse semigroup $S$. Then there is a covering inverse semigroup $T$ containing $S$ as a thick inverse subgroup and an idempotent separating homomorphism $\psi: P(G, \mathscr{X}, \mathscr{Y}) \rightarrow \Gamma(T ; S)$ such tha the diagram

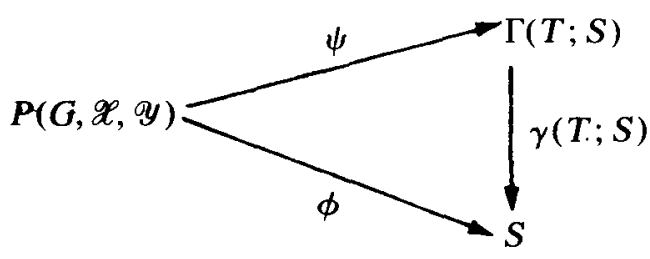

commutes.

Proof. By Theorem 1.4, applied to the semigroup $S^{*}$ of non-zero elements of $\hat{S}$, there is an idempotent separating homomorphism $\phi^{*}: P^{*} \rightarrow S^{*}$. Without loss of generality, we may assume that $P=P(G, \mathscr{X}, \mathscr{Y})$ where $\mathscr{X}$ is a partially ordered set with $\mathscr{Y}$ an essential ideal and subsemilattice and where $G$ acts on $\mathscr{Z}$ by order automorphisms in such a way that $\mathscr{X}=G \cdot \mathscr{Y}$. Then, by Proposition 2.7, $P^{*}=P\left(G, \mathscr{X}^{*}, \mathscr{Y}^{*}\right)$ where $\mathscr{X}^{*}$ is the semilattice of non-empty order ideals of $\mathscr{X}$. Further, by Lemma 5.8, $\rho=\phi^{*} \circ \phi^{*-1}$ can be extended to an idempotent separating congruence $\bar{\rho}$ on $P\left(G, \mathscr{X}^{*}, \mathscr{X}^{*}\right)$. Let $T=P\left(G, \mathscr{X}^{*}, \mathscr{X}^{*}\right) / \bar{\rho}$. Then, Lemmas 5.11 and 5.9 show that $S$ is a thick inverse subsemigroup of $T$ and, as in the proof of Proposition 5.10, $\phi$ factors through $\gamma(T ; S)$.

The results of this section can be put in a more global setting if we introduce two categories; the category $\mathscr{C} \mathscr{C}(s)$ of covering extensions of an inverse semigroup $S$ and the category $\mathscr{P} \mathscr{C}(S)$ of E-unitary coverings of $S$. An object in $\mathscr{C} \mathscr{E}(S)$ is an embedding of $S$ as a thick inverse subsemigroup in a covering inverse semigroup $T$. A morphism between embeddings of $S$ in $T$ and $S$ in $U$ is an idempotent separating homomorphism $\theta: T \rightarrow U$ such that the diagram

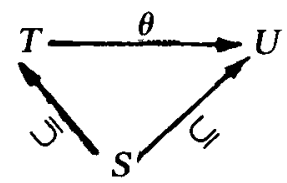

commutes.

An $E$-unitary covering of $S$ is an idempotent separating homomorphism $\phi$ of an $E$-unitary inverse semigroup $P$ onto $S$. A morphism form $\theta: P \rightarrow S$ to 
$\phi: Q \rightarrow S$ is an (idempotent separating) homomorphism $\psi: P \rightarrow Q$ such that the diagram

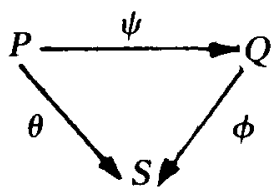

commutes.

THEOREM 5.13. Let $S$ be an inverse semigroup. Then the mapping, which associates with each $T \in \mathscr{C} \mathscr{E}(S)$ the E-unitary inverse semigroup $\Gamma(T ; S)$ the idempotent separating homomorphism $\gamma(T ; S): \Gamma(T ; S) \rightarrow S$ and which associates with $\theta: T \rightarrow U$ in $\mathscr{C} \mathscr{E}(S)$ the homomorphism $\Gamma(\theta)$ restricted to $\Gamma(T ; S) \rightarrow S$ is a functor from $\mathscr{C} \mathscr{E}(S)$ onto a cofinal subcategory of $\mathscr{P} \mathscr{C}(S)$.

ProOf. The only part of the theorem which still requires justification is that $\Gamma(\theta): \Gamma(T) \rightarrow \Gamma(U)$ maps $\Gamma(T ; S)$ into $\Gamma(U ; S)$. This is straightforward.

Similarly, Proposition 5.10 shows that an analogous result holds for coverings $P(G, \mathscr{X}, \mathscr{Y})$ of $S$ with $\mathscr{X}$ a semilattice and $\mathscr{Y}$ an ideal of $\mathscr{X}$.

\section{Examples}

Example 6.1. Brandt Semigroups

In this example, we describe the various constructions of Sections 4 and 5 for a Brandt semigroup $S=\mathscr{M}^{\circ}(G ; I, I ; \Delta)$ with $|I| \geqq 2$. These can be used to show that $\mathscr{P} \mathscr{C}(S)$ does not have a final object.

\subsection{1. $S$ is a covering semigroup.}

We show that $\gamma_{S}: \Gamma(S) \rightarrow S$ is onto. It is shown in Petrich (1973), page 165 that $\Sigma(S)$ is the wreath product $G$ wr $S_{I}$ of $G$ by the group $S_{I}$ of all permutations on $I$. It is easy to see that, for each $e=(i, 1, i)$ in $S$, and each $\alpha=(f, \gamma) \in G$ wr $S_{I}$, $\alpha \cdot e=\left(i \gamma^{-1}, 1, i \gamma^{-1}\right)$. Thus, if we identify the idempotents of $S$ with $I \cup\{0\}$, $G$ wr $S_{I}$ acts on $I^{0}=I \cup\{0\}$ by $\alpha \cdot i=i \gamma^{-1}, \alpha \cdot 0=0$ for $\alpha=(f, \gamma)$. Then $\Gamma(S) \approx$ $P\left(G\right.$ wr $\left.S_{1}: I^{0}, I^{0}\right)$; the homomorphism $\gamma_{S}$ is given by

$$
\begin{aligned}
& (i,(f, \gamma)) \gamma_{s}=(i, f(i), i \gamma) \\
& (0,(f, \gamma)) \gamma_{s}=0 .
\end{aligned}
$$

It is onto, since for each $(i, g, j) \in S$,

$$
(i, g, j)=(i,(f, \gamma)) \gamma_{s}
$$

where $f(k)=g$ for all $k \in I$ and $\gamma$ is the transposition $(i, j)$. 


\subsubsection{The category $\mathscr{P} \mathscr{C}(S)$.}

Suppose that $P$ is an $E$-unitary inverse semigroup and that $\phi$ is an idempotent separating homomorphism of $P$ onto $S$. Then, since $\phi$ is idempotent separating, $P$ and $S$ have "the same" ideal structure. Thus $P$ has a kernel $K$ and $P / K$ is a Brandt semigroup. Hence, from the construction for $E$-unitary inverse semigroups in McAlister (1974a), $P \approx P\left(K, K / H \cup\{K\},\left\{H x_{i}: i \in I\right\} \cup\{K\}\right)$ where $H$ is a subgroup of $K$ and $\left\{x_{i}: i \in I\right\}$ is a set of elements, modulo $H$, such that the costs $H x_{i}$ are in one-to-one correspondence with $I$, with $e \in\left\{x_{i}: i \in I\right\}$. The partial order is determined by $H x>K$ for each $x \in K$ and $K$ acts by $g \cdot H x=H_{x g^{-1}}, g \cdot K=K$. The homomorphism $\phi$ gives rise to a homomorphism $\psi$ of $H$ onto $G$ and $\phi$ is given by

$$
\begin{aligned}
\left(H x_{i}, g\right) \phi & =(i, h, j) \text { where } H x_{i} g=H x_{i} \text { and } h=\left(x_{i} g x_{,}^{-1}\right) \psi \\
(K, g) \phi & =0 .
\end{aligned}
$$

Thus, up to isomorphism, $P$ is determined by a group $K$, a subgroup $H$ of $K$ such that $|K / H| \geqq I$, and a homomorphism $\phi$ of $H$ onto $G$. Conversely, given such $K, H, \phi$ we can construct $P=P\left(K, K / H \cup\{K\},\left\{H x_{i} ; i \in I\right\} \cup\{K\}\right)$ and an idempotent separating homomorphism $\phi$ of $P$ onto $S$.

The semigroup $T$ obtained by extending $\phi{ }^{\circ} \phi^{-1}$ to an idempotent separating congruence on $P(K, K / H \cup\{K\}, K / H \cup\{K\})$ is $\mathcal{M}^{0}(G ; K / H, K / H ; \Delta)$ and $\Gamma(T ; S)$ is $P\left(G\right.$ wr $\left.S_{K / H}, K / H^{0}, I^{0}\right)$ where $I$ is embedded in $K / H$ by a choice of coset representatives; $\Gamma(T ; S)$ is independent of the particular embedding of $I$ into $K / H$.

REMARK. The covering semigroups $T$ which arise in 5.1.2 are the semigroups $\mathcal{M}^{0}(G ; J, J ; \Delta)$ where $|J| \geqq|I|$. There are many covering semigroups which contain $S$ as a heavy subsemigroup. However, if $U$ is a covering semigroup which contains an inverse semigroup $S$ as a heavy subsemigroup, then $T=\left\{a \in U: a \mathscr{D}_{U} x\right.$ for some $\left.x \in S\right\}$ is also a covering semigroup and $\Gamma(U ; S)=\Gamma(T ; S)$. Thus, without loss of generality it suffices to consider covering semigroups $U$ containing $S$ so that $S$ meets each $\mathscr{D}$-class of $U$. For Brandt semigroups, these are precisely the semigroups $\mathcal{M}^{0}(G ; J, J ; \Delta)$ with $|\boldsymbol{J}| \geqq|I|$.

\subsubsection{The category $\mathscr{P} \mathscr{C}(S)$ does not have a final object.}

Let $J$ be any set, containing $I$, with $|J| \geqq \max \{|I|, 5\}$; then the alternating group $A_{J}$ is simple and acts transitively on $J$. Let $K=G \times A_{J}$. Then $K$ acts on $J^{0}$ by $(g, \alpha) \cdot j=j \alpha^{-1},(g, \alpha) \cdot 0=0$. Since $J^{0}$ is a semilattice, under $j>0$ for each $j \in J$, containing $I^{0}$ as an ideal, we can form $P\left(K, J^{0}, I^{0}\right)$. The mapping $\pi_{J}: P\left(K, J^{0}, I^{0}\right) \rightarrow S$ defined by 


$$
\begin{aligned}
& (i,(g, \alpha)) \pi_{J}=(i, g, i \alpha) \\
& (0,(g, \alpha)) \pi_{J}=0
\end{aligned}
$$

is then an idempotent separating homomorphism of $P\left(K, J^{0}, J^{0}\right)$ onto $S$.

Suppose that $\phi: P(H, \mathscr{Q}, \mathscr{Y}) \rightarrow S$ is a final object in $\mathscr{P} \mathscr{C}(S)$. Then there is a homomorphism $\psi: P\left(K, J^{0}, I^{0}\right) \rightarrow P(H, \mathscr{X}, \mathscr{Y})$ such that $\psi \phi=\pi$. By McAlister (1974a), $\psi$ gives rise to a homomorphism $\psi_{1}$ of $K$ into $H$ and an isotone mapping $\psi_{2}$ of $J^{0}$ into $\mathscr{X}$ such that

$$
(i,(g, \alpha)) \psi=\left(i \psi_{2},(g, \alpha) \psi_{1}\right)
$$

and

$$
[(g, \alpha) \cdot i] \psi_{2}=(g, \alpha) \psi_{1} \cdot i \psi_{2} \text {. }
$$

Since $A_{J}$ is simple, either $\psi_{1}$ is one-to-one on $\{1\} \times A_{J}$ or is trivial on $\{1\} \times A_{J}$. But, since $(g, \alpha) \cdot i=i \alpha^{-1}$, the latter implies $(i \alpha) \psi_{2}=i \psi_{2}$ for each $i \in I$. This means that $P\left(K, J^{0}, J^{0}\right) \psi$ is a union of groups; $(i,(g, \alpha)) \psi$ belongs to the subgroup with identity $\left(i \psi_{2}, 1\right)$. But then $\psi \phi=\pi_{J}$ also implies $S$ is a union of groups which is a contradiction. Hence $\psi_{1}$ is one-to-one and $|K| \geqq\left|A_{J}\right| \mid$ for each $J$. This is impossible.

EXAMPLE 6.2. Let $G$ be any group and let $\mathscr{X}=G^{0}, \mathscr{y}=\{1,0\}$ with $x>0$ for all $x \in G$. Then $\mathscr{X}$ is a semilattice and $G$ acts on $\mathscr{X}$ by $g \cdot x=x g^{-1}$. The $E$-unitary inverse semigroup $P=P(G, \mathscr{X}, \mathscr{Y})$ is essentially $G$ with an extra identity adjoined. Thus there is an idempotent separating homomorphism $\phi$ of $P$ onto $\{1,0\}=P\left(\{1\},\{1\}^{0},\{1\}^{0}\right)$; it is given by $(1,1) \phi=1,(0, g) \phi=0$. The homomorphism $\phi$ cannot be extended to a homomorphism of $P(G, \mathscr{X}, \mathscr{X})$ into $\{1,0\}$; the latter is already in the form $P(H, U, U)$. The inverse semigroup $T$ corresponding to $\phi$ and $P$ is $\mathcal{M}^{\circ}(\{1\} ; G, G ; \Delta)$ which contains $\{1,0\}$ as a heavy inverse subsemigroup.

The procedure which underlies the results of Section 5 can be used to analyse the structure of inverse semigroups which arise in several concrete situations. We give two examples.

EXAMPLE 6.3. The semigroup of relative isomorphisms of a field extension.

Let $K$ and $F$ be fields with $K$ algebraic over $F$. Then a relative isomorphism of $K$ over $F$ is an $F$-algebra isomorphism of a subfield of $K$ onto a subfield of $K$. Thus it is an element of $\mathscr{I}_{K}$. The set of relative isomorphisms is clearly closed under composition and inverses so that it is an inverse subsemigroup of $\mathscr{I}_{K}$; we shall denote this semigroup by $\mathscr{G}(K ; F)$, the Galois semigroup of $K$ over $F$.

The idempotents of $\mathscr{G}(K, F)$ are the identity maps on the subfields of $K$ which contain $F$. Thus they form a semilattice isomorphic to the semilattice $\mathscr{K}$ of 
subfields of $K$ which contain $F$. If $L$ is such a subfield, then the maximal subgroup of $\mathscr{G}(K, F)$ corresponding to $L$ is exactly the Galois group $G(L, F)$ of $L$ over $F$.

Since $(K, F)$ has an identity, $\Sigma(\mathscr{G}(K, F)) \approx G(K, F)$ and so $\Gamma(\mathscr{G}(K, F)) \approx$ $P(G(K, F), \mathscr{K}, \mathscr{K})$ where $\mathscr{K}$ denotes the semilattice of subfields of $K$, which contains $F$, and where $G(K, F)$ acts on $\mathscr{K}$ by $\alpha \cdot L=L \alpha^{-1}$ for each $\alpha \in G(K, F)$. Further $(L, \alpha) \gamma_{T}=\left.\alpha\right|_{L}$ for each $(L, \alpha) \in \Gamma(T)$ with $T=\mathscr{G}(K, F)$. Hence $\mathscr{G}(K, F)$ is a covering semigroup if and only if each relative isomorphism of $K$ over $F$ can be extended to an automorphism of $K$ over $F$. In particular $\mathscr{G}(K, F)$ is a covering semigroup if $K$ is normal over $F$.

Suppose that $A$ is normal extension of $F$ and contains $K$. Then $\mathscr{G}(A, F)=T$ is a covering inverse semigroup containing $S=\mathscr{G}(K, F)$ as a heavy subsemigroup. Thus $\Gamma(T ; S)$ is a proper inverse semigroup and $\gamma(T ; S)$ is an idempotent separating homomorphism of $\Gamma(T ; S)$ onto $S$. From the preceding paragraph, $\Gamma(T ; S)=P(G(A, F), \mathscr{A}, \mathscr{K})$ where $\mathscr{A}$ is the set of subfields of $A$ containing $F$ and $\mathscr{K}$ is the set of subfields of $K$ that contain $F$. Further, for each $(L, \alpha) \in$ $\Gamma(T ; S),(L, \alpha) \gamma(T ; S)=\left.\alpha\right|_{L}$. The maximal subgroup of $\Gamma(T ; S)$ containing $(L, 1)$ is isomorphic to the stabilizer $H_{L}$ of $L$ under $G(A, F)$; the kernel $\{\alpha \in G(A, F):(L, \alpha) \gamma(T ; S)=(L, 1) \gamma(T ; S)\}$ is $G(A, L)$. Hence $H_{L} / G(A, L) \approx$ $G(L, F)$. In particular, if $L$ is normal so that $H_{L}=G(A, F)$ we have $G(A, F) / G(A, L) \approx G(L, F)$.

\section{EXAMPLE 6.4. Isomorphisms of cyclic subgroups of a group.}

Let $G$ be a group. Then, since the intersection of cyclic subgroups of $G$ is cyclic, it is easy to see that the set of isomorphisms between cyclic subgroups of $G$ is an inverse subsemigroup of $\mathscr{I}_{G}$; we shall denote this semigroup $\mathscr{C}(G)$.

The semigroup $\mathscr{C}(G)$ is 0 -bisimple if and only if any two nonidentity elements of $G$ have the same order. Thus $\mathscr{C}(G)$ is 0 -bisimple if and only if either $G$ is torsion free or has exponent $p$ for some prime $p$. In the first case $\mathscr{C}(G)$ has infinite chains of idempotents; in the second it is a Brandt semigroup.

We shall concentrate on the case when $G$ is a finite abelian group. By the structure theorem for finite abelian groups $G \approx P_{1} \times P_{2} \times \cdots \times P_{r}$, where $P_{1}, \cdots, P_{r}$ are $p_{i}$-primary groups for distinct primes $p_{1}, \cdots, p_{r}$. It is easy to see that $\mathscr{C}(P) \approx \mathscr{C}\left(P_{1}\right) \times \cdots \times \mathscr{C}\left(P_{r}\right)$ so that, in order to calculate $\mathscr{C}(G)$ it suffices to consider $G p$-primary for some prime $p$.

If $G$ is embedded in a $p$-primary abelian group $A$ which has the property that, each $\alpha \in \mathscr{C}(A)$ can be extended to an automorphism of $A$, then we can apply the ideas of Section 5 to get a proper covering for $\mathscr{C}(G)$. Let $\mathscr{Z}$ be the semilattice of cyclic subgroups of $A$ and $\mathscr{Y}$ that of $G ; \mathscr{Y}$ is an ideal of $\mathscr{X}$. Then Aut $A$ acts on $\mathscr{X}$ by $\alpha \cdot\langle x\rangle=\left\langle x \alpha^{-1}\right\rangle$ for each $x \in A, \alpha \in$ Aut $A$ so we can construct $P$ (Aut $A, \mathscr{X}, \mathscr{Y}$ ). The mapping $\eta$ given by $(\langle x\rangle, \alpha) \eta=\left.\alpha\right|_{\langle x\rangle}$ is then an idempotent separating homomorphism of $P=P$ (Aut $A, \mathscr{X}, \mathscr{Y}$ ) onto $\mathscr{C}(G)$. 
Simple group theory shows every $\alpha \in \mathscr{C}(A)$ can be extended to an automorphism of $A$ if and only if $A$ if and only if $A$ is a direct product of cyclic groups of the same prime power order. Hence if $G \approx \mathbf{Z}_{p^{\alpha_{1}}} \times \cdots \times \mathbf{Z}_{p} \boldsymbol{\alpha}_{n}$ where $\alpha_{1} \leqq \alpha_{2} \leqq \alpha_{n}$ we can take $A$ to be the direct product of $n$ copies of $Z_{p} \alpha_{n}$. The automorphism group of $A$ is then $G L\left(Z_{p^{\alpha_{n}}}\right)$ so that $P$ can be calculated directly.

\section{References}

J. E. Ault (1972), 'Translational hull of an inverse semigroup', Semigroup Forum 4, 165-168.

S. Y. Chen and S. C. Hseih (1974), 'Factorizable inverse semigroups', Semigroup Forum 8, 283-297.

A. H. Clifford and G. B. Preston (1961), Algebraic Theory of Semigroups, Vol. 1, Math Surveys 7, (American Math. Soc., Providence, R. I.)

D. B. McAlister (1974),'Groups, semilattices and inverse semigroups', Trans. American Math. Soc. 192, 227-244.

D. B. McAlister (1974a), 'Groups, semilattices and inverse semigroups, II', Trans. American Math. Soc. 196, 351-370.

D. B. McAlister (1975), 'One-to-one partial right translations of a right cancellative semigroup', to appear J. Algebra.

R. McFadden and L. O'Carroll (1971), 'F-inverse semigroups', Proc. London Math. Soc. (3) 22, $652-666$.

L. O'Carroll (1974), 'Reduced inverse and partially ordered semigroups', J. London Math. Soc. (2) 293-301.

L. O'Carroll (1975), 'Embedding theorems for proper inverse semigroups', submitted to $J$. Algebra.

M. Petrich (1973), Introduction to Semigroups, Charles E. Merrill Publishing Company.

N. R. Reilly (1974), 'Extension of congruences and homomorphisms to translational hulls', Pacific $J$. Math. 54, 209-228.

T. Saito (1965), 'Proper ordered inverse semigroups', Pacific J. Math. 15, 649-666.

B. M. Schein (1973), 'Completions, translational hulls and ideal extensions of inverse semigroups', Czechosiovak Math. J. 575-609.

\section{Department of Mathematical Sciences}

Northern Illinois University

DeKalb, Illinois 60115

U.S.A. 